\title{
Dexamethasone blocks the migration of the human neuroblastoma cell line SK-N-SH
}

L.A. Casulari ${ }^{1}$, D. Dondi ${ }^{2}$, R. Maggi ${ }^{2}$, M. Demissie ${ }^{3}$, M. Piccolella ${ }^{2}$ and F. Piva ${ }^{2}$

\author{
${ }^{1}$ Escola Superior em Ciências da Saúde, Brasília, DF, Brasil \\ ${ }^{2}$ Department of Endocrinology, Center of Endocrinological Oncology, \\ University of Milano, Milano, Italy \\ ${ }^{3}$ Department of Endocrinology and Diabetology, Wroclaw Medical University, \\ Wroclaw, Poland
}

\section{Correspondence}

D. Dondi

Institute of Endocrinology

Center of Endocrinological Oncology

University of Milano

Via Balzaretti, 9

20133 Milano

Italy

Fax: +39-02-5031-8204

E-mail: dondilab@unimi.it

Received July 11, 2005

Accepted May 29, 2006

\begin{abstract}
Glucocorticoids (Gc) influence the differentiation of neural crestderived cells such as those composing sympathoadrenal tumors like pheochromocytomas, as well as neuroblastomas and gangliomas. In order to obtain further information on the effects of Gc on cells evolving from the neural crest, we have used the human neuroblastoma cell line SK-N-SH to analyze: 1) the presence and the binding characteristics of Gc receptors in these cells, 2) the effect of dexamethasone (Dex) on the migration of SK-N-SH cells, and 3) the effect of Dex on the organization of the cytoskeleton of SK-N-SH cells. We show that: 1 ) receptors that bind $\left[{ }^{3} \mathrm{H}\right]$-Dex with high affinity and high capacity ( $\mathrm{Kd}$ of $9.6 \mathrm{nM}$, Bmax of $47 \mathrm{fmol} / \mathrm{mg}$ cytosolic protein, corresponding to 28,303 sites/cell) are present in cytosolic preparations of SK-N-SH cells, and 2) treatment with Dex (in the range of 10 $\mathrm{nM}$ to $1 \mu \mathrm{M}$ ) has an inhibitory effect (from $100 \%$ to 74 and $43 \%$, respectively) on the chemotaxis of SK-N-SH cells elicited by fetal bovine serum. This inhibition is completely reversed by the Gc receptor antagonist RU486 (1 $\mu \mathrm{M})$, and 3) as demonstrated by fluorescent phalloidin-actin detection, the effect of Dex (100 nM) on SK-N$\mathrm{SH}$ cell migration is accompanied by modifications of the cytoskeleton organization that appear with stress fibers. These modifications did not take place in the presence of $1 \mu \mathrm{M}$ RU486. The present data demonstrate for the first time that Dex affects the migration of neuroblastoma cells as well as their cytoskeleton organization by interacting with specific receptors. These findings provide new insights on the mechanism(s) of action of Gc on cells originating in the neural crest.
\end{abstract}

\section{Introduction}

Differentiation of the cells derived from the neural crest is strongly influenced by the endocrine environment (1-7). The study of
- Human neuroblastoma

- SK-N-SH cells

- Glucocorticoids

- Migration

- Cytoskeleton
Key words the influence of hormones on cell differentiation was facilitated after different cell lines were isolated from tumors derived from cells originating in the neural crest (neuroblastomas, pheochromocytomas). 
Neuroblastomas are neuroendocrine solid tumors of childhood that show undifferentiated cell phenotypes and a poor prognosis. Human neuroblastoma cell lines correspond to sympathoadrenal neuroblasts, arrested at different levels during embryogenesis $(5,8)$. They retain the capacity to evolve to different kinds of cells according to the environmental signals. The differentiation of sympathoadrenal precursor cells may proceed towards either sympathetic neurons or chromaffin cells; these changes may be induced by several factors and hormones. Neuroblastoma cells have been consequently utilized as a model to analyze the effects of the endocrine milieu on the differentiation of sympathoadrenal cells $(6,7,9,10)$.

Several studies suggest that glucocorticoids $(\mathrm{Gc})$ have a potent effect inducing the differentiation of neural crest cells towards the chromaffin cell type of the adrenal medulla (7,10-15). In addition, Gc influence a series of biochemical parameters of neuroblastoma cells that may further suggest a possible differentiation of these cells. Gc can potentiate tyrosine hydroxylase activity in mouse NBP2 neuroblastoma cells (16-19) and induce the prostaglandin E1-mediated cyclic AMP formation in murine N1E-115 neuroblastoma cells $(20,21)$. The expression of two markers of neuronal/neuroendocrine development, pG8 and pG2, was induced by the glucocorticoid agonist dexamethasone (Dex) in LA-N-5 neuroblastoma cells (22, 23). Finally, Gc can induce cyclooxygenase1 expression in NG108 and N2a neuroblastoma cells $(24,25)$. It has also been reported that Dex induces a chromaffin phenotype, an increase in the cell content of chromogranin A, thyrosine hydroxylase and a decrease of secretogranin II and neurofilaments in a series of neuroblastoma cells. These effects are blocked by the Gc antagonist RU486 (11).

No data are available on the motility of neuroblastoma cells under proper stimuli and on the possible effects of Gc on this cellular activity. New information on these issues could provide a useful tool for the evaluation of the invasive potential of neuroblastomas. One may hypothesize that changes of motility under the effect of Gc imply a rearrangement of cytoskeleton organization; indeed, reports have been published indicating that Gc affect the morphology and cytoskeleton organization of different cell types $(8,9)$.

The effects of Gc on neuroblastoma cell lines imply that Gc receptors (GR) are present in neuroblastoma cells; one may conclude that cytoplasmic GR upon interaction with cell DNA bring about a series of genomic modifications leading to morphological and biochemical modifications as per the classical mechanism of action of Gc (for a review, see Ref. 26) which are involved in essential biological processes such as growth, development, metabolism, behavior, and apoptosis. Only one report has been published so far indicating, on the basis of Western blot analysis, that GR are present in several neuroblastoma cell lines (6). No study has been devoted to the analysis of the binding characteristics of GR present in neuroblastoma cells. To obtain some insights on the binding characteristics of GR and on the effects of Gc on neuroblastoma cell migration and cytoskeleton organization, experiments have been performed with the use of the human neuroblastoma cell line SK-N$\mathrm{SH}$, a cell line isolated from a bone marrow metastasis of a 4-year-old girl, that has been frequently used in this kind of study since SK-N-SH cells mimic many of the properties of neural crest cells $(10,27)$. SK-N-SH cells are composed of at least three kinds of phenotypically different cells, the neuroblastlike cells ( $85 \%$ of the total), the cells expressing some features of the glial and epithelial cells (5\% of the whole population), or chromaffin cells, and the cells expressing an intermediate phenotype between neuroblastand epithelial-like cells (10\% of the total) that still retain the capacity to transdifferen- 
tiate under proper stimuli (7,10,14,27-29).

The experiments reported here have been performed with the use of Dex, the prototypic synthetic Gc, and analyzed: 1) the presence and the binding characteristics of GR in SK$\mathrm{N}-\mathrm{SH}$ cells, 2) the effect of Dex on the migratory activity of SK-N-SH cells, and 3) whether changes in motility are accompanied by some structural and morphological modification of the cells under investigation, as evaluated by the morphological analysis of cytoskeleton organization and cell shape.

\section{Material and Methods}

\section{Cell cultures}

SK-N-SH cells, obtained from the American Type Culture Collection (Rockville, MD, USA), were routinely grown in DMEM (Biochrom KG, Berlin, Germany) supplemented with $10 \%$ fetal bovine serum (FBS, Gibco, Paisley, Scotland, UK), glutamine (1 mM), and antibiotics (100 units/mL penicillin $\mathrm{G}$ sodium, $100 \mathrm{mg} / \mathrm{mL}$ streptomycin sulfate) in a humidified atmosphere of $5 \% \mathrm{CO}_{2}: 95 \%$ air at $37^{\circ} \mathrm{C}$. Dex and RU486, obtained from Sigma (Milan, Italy), were dissolved in ethanol. The final concentration of ethanol in the culture medium did not exceed $0.01 \%$.

\section{Binding studies}

Cells plated onto $10 \%$ charcoal-stripped FBS (CS-FBS)/DMEM culture medium were collected and homogenized in TEDG buffer (10 mM Tris-HCl, pH 7.5, 1 mM EDTA, 2 $\mathrm{mM}$ dithiothreitol, $10 \%$ glycerol, $5 \mu \mathrm{g} / \mathrm{mL}$ antipain and leupeptin) and centrifuged at $110,000 \mathrm{~g}$ to obtain the cytosolic fraction. Protein concentration was measured by the method of Bradford (30). The binding assay was performed using $\left[{ }^{3} \mathrm{H}\right]$-Dex (S.A.: 82.0 $\mathrm{mCi} /$ umol; Amersham Biosciences, Buckinghamshire, UK) as specific GR ligand in the presence of increasing concentrations (5 $\mathrm{x} 100 \mathrm{nM}$ to $5 \mathrm{x} 10 \mu \mathrm{M}$ ) of Dex. Incubation was carried out at $4^{\circ} \mathrm{C}$ and the unbound (1) Dex was removed by absorption with dextran-coated charcoal ( $0.5 \%$ Norit A, $0.05 \%$ dextran, $10 \mathrm{mM}$ Tris, and $1 \mathrm{mM}$ EDTA, $\mathrm{pH}$ 7.5). The radioactivity of $\left[{ }^{3} \mathrm{H}\right]-\mathrm{Dex}$ bound to GR was measured in a counter for $B$ emitters (Tri Carb 1600 CA, Liquid Scintillation Analyzer, Packard, Milano, Italy). The binding parameters $(\mathrm{Kd}$, dissociation constant; Bmax, maximal binding capacity) for GR were obtained from the analysis of homologous competition curves using the LIGAND computer program (10).

\section{Microchemotaxis assay}

Subconfluent SK-N-SH cells, plated onto $10 \%$ CS-FBS/DMEM culture medium, were treated with graded doses $(0.1 \mathrm{nM}$ to $1 \mu \mathrm{M})$ of Dex every other day for 6 days. To test the specificity of the effect of Dex, SK-N-SH cells were treated every other day for 6 days with either Dex alone (100 nM) or with Dex in the presence of the GR antagonist RU486 $(1 \mu \mathrm{M})$. The results were compared with those obtained in cells treated only with ethanol $(0.01 \%)$ that was used as the vehicle for the steroids under study.

The microchemotaxis assay was performed as described by Maggi et al. (21). Briefly, cell migration tests were performed using a 48-well Boyden chamber (Neuroprobe, Cabin John, MD, USA) having a lower and an upper compartment. A polycarbonate filter of $8-\mu \mathrm{m}$ pore size was coated with gelatin and positioned between the two compartments; the lower compartment was loaded with FBS as the chemotactic agent. The cell suspensions $(100,000$ cells/50 $\mu \mathrm{L})$ were placed in the upper compartment and incubated for $4 \mathrm{~h}$ at $37^{\circ} \mathrm{C}$. Then, the membrane was scraped-off and the cells adherent to the lower surface were stained with Diffquik (Biomap, Como, Italy) and counted by light microscopy at $1000 \mathrm{X}$ magnifications. Six random objective fields of stained 
cells were counted for each well and the mean number of migrating cells $/ \mathrm{mm}^{2}$ was calculated. Each sample was studied in 12 replicates and each experiment was repeated three times. Data were analyzed statistically by one-way analysis of variance followed by the Dunnett test (23). Data are reported as means \pm SEM.

\section{Fluorescent phalloidin-actin detection}

Subconfluent SK-N-SH cells, plated onto $10 \%$ CS-FBS/DMEM culture medium, were treated every other day for 6 days with either Dex alone $(100 \mathrm{nM})$ or with Dex in the presence of the GR antagonist RU486 (1 $\mu \mathrm{M})$. The organization of the cytoskeleton of SK-N-SH cells was analyzed by the use of phalloidin. Briefly, after the treatments, SK$\mathrm{N}$-SH cells were plated onto 24-multiwell

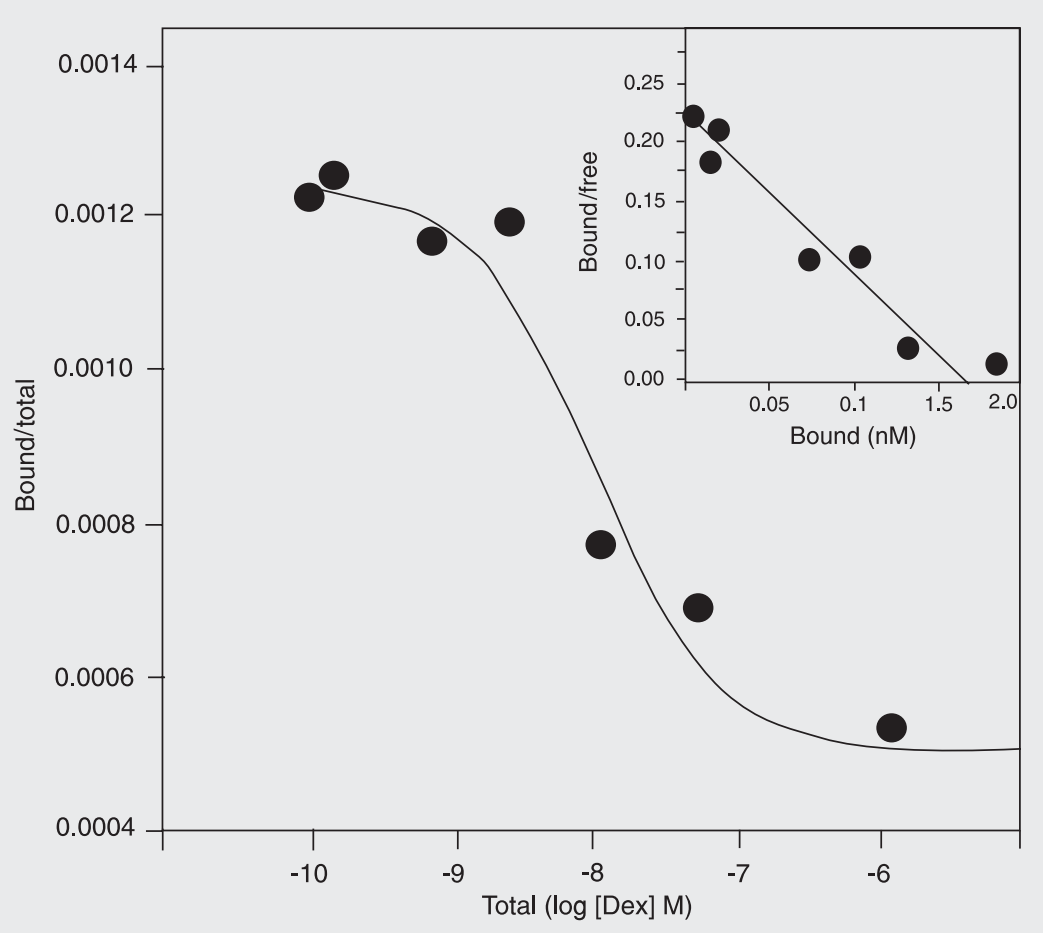

Figure 1. Binding of $\left[{ }^{3} \mathrm{H}\right]$-dexamethasone $\left(\left[{ }^{3} \mathrm{H}\right]\right.$-Dex) to SK-N-SH neuroblastoma cell cytosolic preparations. The isotherms represent the LIGAND-generated curves (untransformed data) or the Scatchard plot (inset) of the total binding of $\left[{ }^{3} \mathrm{H}\right]$-Dex. The calculated binding parameters are $\mathrm{Kd}$ of $9.6 \mathrm{nM}$ and Bmax of $47 \mathrm{fmol} / \mathrm{mg}$ cytosolic protein corresponding to 28,303 sites/cell. plates on 10-mm poly-L-lysine coated glasses (100 cells/well in $500 \mu \mathrm{L}$ DMEM medium with $10 \%$ FBS). Cells were fixed in $4 \%$ paraformaldehyde and the cell membranes were permeabilized with $0.1 \%$ Triton $\mathrm{X}$ 100, incubated with FITC-labeled phalloi$\operatorname{din}(0.2 \mu \mathrm{g} / \mathrm{mL}$, Sigma, St. Louis, MO, USA $)$ at $37^{\circ} \mathrm{C}$ for $10 \mathrm{~min}$ and observed under a Zeiss Axiovert microscope. The data obtained were compared to those derived from cells treated with ethanol $(0.01 \%)$ that served as controls.

\section{Results}

The first series of experiments was addressed to detect the presence of GR in SK$\mathrm{N}-\mathrm{SH}$ cells and to analyze the binding characteristics of GR. The results obtained indicated that cytosolic preparations of SK-N$\mathrm{SH}$ cells express GR. Figure 1 illustrates the isotherms of the LIGAND-generated curves (untransformed data) and the Scatchard plot (inset) of the total binding of $\left[{ }^{3} \mathrm{H}\right]$-Dex to cytosolic preparations obtained from SK-N$\mathrm{SH}$ cells. The calculated binding parameters indicate that SK-N-SH cells express a single class of receptors with a $\mathrm{Kd}$ of $9.6 \mathrm{nM}$ and a Bmax of $47 \mathrm{fmol} / \mathrm{mg}$ cytosolic protein, corresponding to 28,303 sites/cell.

Figure 2, panel A, shows that a 6-day exposure of SK-N-SH cells to graded concentrations of Dex (from $0.1 \mathrm{nM}$ to $1 \mu \mathrm{M}$ ) had an inhibitory effect on the chemotaxis of the cells elicited by FBS; the inhibitory effect was significant at concentrations of Dex in the range of $10 \mathrm{nM}$ to $1 \mu \mathrm{M}$.

In a second series of experiments, we tested the specificity of the effect of Dex on the motility of SK-N-SH cells. The presence of the GR antagonist RU486 $(1 \mu \mathrm{M})$ that per se did not have any effect on cell motility totally abolished the inhibitory effect of Dex $(100 \mathrm{nM})$ on the chemomigration of SK-NSH cells (Figure 2, panel B).

Based on these results, the Dex dose of $100 \mathrm{nM}$ was used in the subsequent test, 
since it was found to be the lowest dose able to affect the chemomigration of SK-N-SH cells

In Dex-treated cells a rearrangement of the actin cytoskeleton was observed. Figure 3 shows that in control cells (panel A) Factin was poorly organized, dispersed throughout the cells and more concentrated at the level of membrane ruffles and lamellipodia (arrowheads). After Dex addition, F-actin appeared to be organized in a clear stress fiber network (panel B). The addition of the GR antagonist RU486 alone ( $1 \mu \mathrm{M})$ to SK$\mathrm{N}-\mathrm{SH}$ cells had no effect on the organization of the cytoskeleton (panel C), which maintained ruffles and lamellipodia, but counteracted the effect of Dex (panel D). It seems interesting to note that three days after the removal of Dex, cells still exhibited the features reported in panel B. This suggests that a rather sustained effect was induced by the presence of the drug. Dex and RU486 had an effect also on the morphology of the cells: under Dex treatment neurite-like processes were disappearing and the cells expressed a round flattened shape resembling the aspect of chromaffin epithelial-like cells (panel B). After the addition of RU486 at a dose 10fold higher than Dex, the cells became starlike and flatter than control cells (panel C).

\section{Discussion}

The binding experiments performed on SK-N-SH cell cytosolic preparations show that these cells express a single class of high affinity and high capacity Dex receptors. The binding characteristics recorded are consistent with those reported for GR in cytosolic preparations of brain cells $(25,31)$ and leukocytes (32).

To the authors' knowledge, this is the first report describing the binding characteristics of Dex in a neuroblastoma cell line. This finding confirms and extends previous observations by Glick et al. (6) who demonstrated the presence of GR by Western blot analysis in a series of neuroblastoma cell lines that did not include SK-N-SH cells. As a whole, the findings from this and other laboratories indicate that Gc may act on neuroblastoma cell lines by interacting with

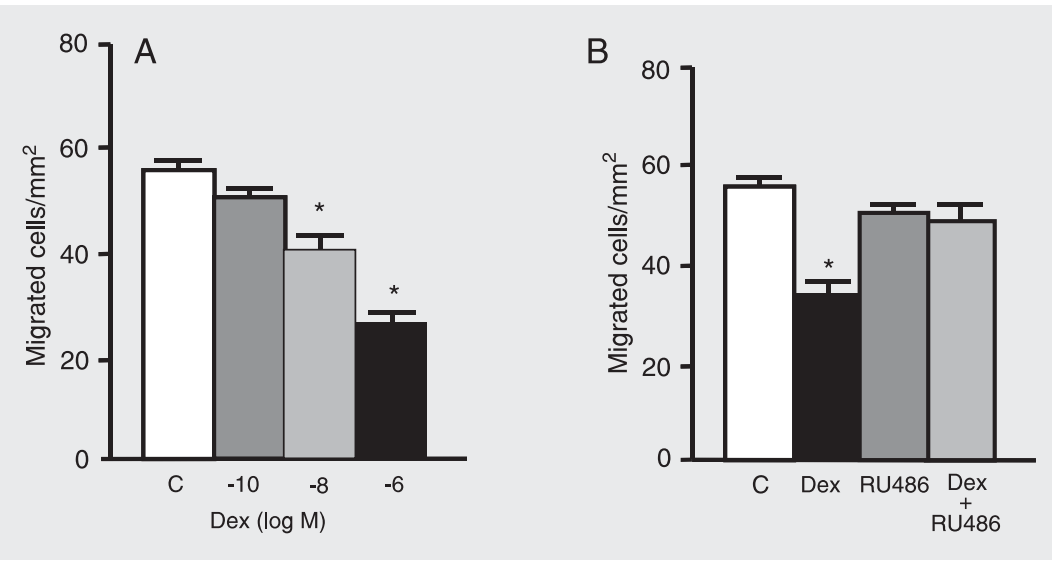

Figure 2. $A$, Effect of dexamethasone (Dex) $(0.1 \mathrm{nM}$ to $1 \mu \mathrm{M})$ on the chemotactic response of SK-N-SH cells to fetal bovine serum. The experiments were performed using the Boyden chamber assay and the treatment was for 6 days. B, Effect of a 6-day treatment with Dex $(100 \mathrm{nM})$ and RU486 $(1 \mu \mathrm{M})$ added separately or in combination on the chemotactic response of SK-N-SH cells to fetal bovine serum. $\mathrm{C}=$ control cells treated with the vehicle only, $0.01 \%$ ethanol. ${ }^{*} \mathrm{P}<0.05$ vs control (Dunnett test).
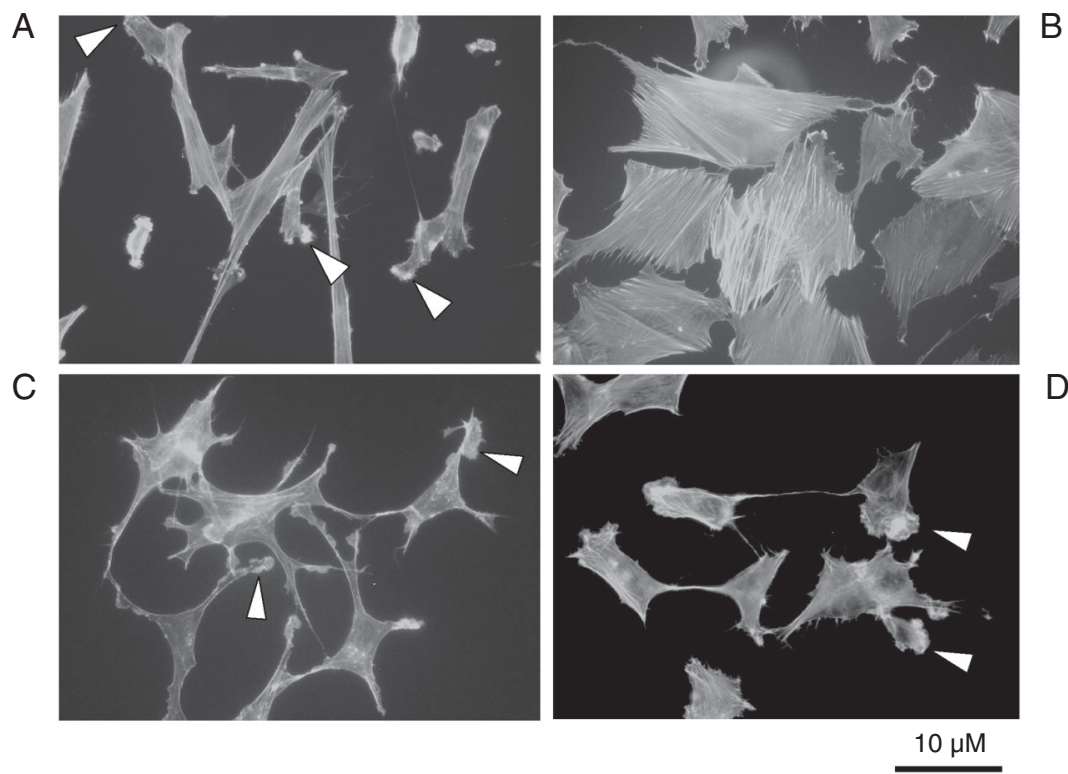

Figure 3. Detection of fluorescent phalloidin-actin in the cytoskeleton of SK-N-SH cells under different experimental conditions. A, SK-N-SH control cells treated with vehicle, $0.01 \%$ ethanol; $B$, SK-N-SH cells treated for 6 days with dexamethasone (Dex) alone (100 $\mathrm{nM})$; $C$, SK-N-SH cells treated for 6 days with the glucocorticoid receptor antagonist RU486 $(1 \mu \mathrm{M}) ; D$, SK-N-SH cells treated with Dex $(100 \mathrm{nM})$ in association with RU486 (1 $\mu \mathrm{M})$. Arrowheads indicate ruffles and lamellipodia. 
specific receptors.

The data presented here show that Dex reduces the chemotactic response of SK-N$\mathrm{SH}$ cells to FBS. The observation that the effect of Dex is abolished by the GR antagonist RU486 further supports the view that Dex acts on neuroblastoma cells through the activation of specific receptors.

The finding that Dex exerts an inhibitory effect on the migratory capacity of SK-N$\mathrm{SH}$ cells agrees with previous reports suggesting that glucocorticoids basically reduce the invasive capability of a variety of tumor cells. For example, it has been reported that Dex decreases the migration rate induced by EGF on HSC-2 cells derived from a buccal mucosa squamous cell carcinoma (27) and that $\mathrm{Gc}$ reduce the motility of eosinophils in nasal polyps (28). These in vitro experiments are reminiscent of in vivo studies demonstrating that Gc exert an inhibitory effect on the migration of neuronal cells during fetal life in mammals $(29,33,34)$. In view of these findings, one may hypothesize that Gc utilized to keep under control a variety of brain tumors (35) exert their effects not only by reducing brain edema and cell proliferation but also by acting on the migratory capacity of tumor cells. This hypothesis is further supported by preliminary results obtained in this laboratory showing that Dex reduces the capability of SK-N-SH cells to migrate into a tridimensional gel matrix in response to FBS (data not shown). This view seems to be further supported by the recent paper by Gut et al. (36). These investigators have reported that in mice deficient for the nuclear orphan receptor steroidogenic factor 1 and lacking adrenal cortical cells and gonads the "adrenal" region exhibits the presence of sympathoadrenal cell precursors as early as on day 12.5 of embryonic life. This may mean that the absence of glucocorticoids favors the migration and/or colonization of sympathoadrenal precursors in the adrenal anlage.

In addition to the inhibitory effect on cell migration, Dex also induced a rearrangement of the cytoskeleton of SK-N-SH cells; similarly Romero et al. (9) have reported that Dex treatment induces changes in the cytoskeleton of cultured rat brain endothelial cells. This effect of Dex has been confirmed by in vivo studies showing that prenatal exposure to $\mathrm{Gc}$ brings about modifications of the cell cytoskeleton in the fetal baboon brain (8). Since the cytoskeleton provides "a structural framework around which cell shape and polarity are defined" (37) it is tempting to conclude that changes in the cytoskeleton organization are involved in the modification of the cell migration induced by Dex. On the other hand, it has been reported that Gc induce morphological changes of neuroblastoma cells $(6,7,14)$. The results reported here showing that Dex treatment brings about a transdifferentiation of SK-N-SH cells that shift from the neurallike to the epithelial-like phenotype seem to confirm these findings. The effect induced was rather sustained, since it was still present three days after the removal of Dex. This might mean that a genomic effect was taking place leading to a permanent rearrangement of actin that could be at the basis of the migration blockade. These results seem to confirm those of Ross et al. (11) who also reported that under Dex treatment neuroblastoma cells were assuming a chromaffinlike aspect. Even though we did not analyze whether these cells were also exhibiting some biochemical features of epithelial-like cells their general aspect could lead to this conclusion, in agreement with several in vitro and in vivo results suggesting that Gc may induce a transdifferentiation of neuroblastoma cells towards a chromaffin, epitheliallike phenotype $(7,10,12-15)$. The results reported here indicate that RU486, while counteracting the effect of Dex on chemomigration, induced a modification of the morphology of the cells. This suggests that the Gc antagonist may have specific actions on the morphology of SK-N-SH cells, possibly act- 
ing through one of the GR isoforms now evidenced in Gc target tissues (26), that could explain the partial agonistic activity displayed by RU486 on some occasions. It may be concluded that Gc may affect the morphology and motility of neuroblastoma cells through modifications of the cytoskeleton organization. Even if our results do not provide a clear cut demonstration of a direct effect of Gc on actin remodeling, several literature data suggest this possibility: it has been reported that Dex stimulates a rapid polymerization of actin and stabilization of microfilaments in human endometrial adenocarcinoma cells (38), that Gc may modify F-actin distribution in a number of cell types $(38,39)$ and that Dex induces morphological differentiation and actin reorganization of human trabecular meshwork tissue after 1014 days of treatment (40).

It may be concluded that Gc may affect the morphology and the motility of neuroblastoma cells through modifications of their cytoskeleton.

\section{References}

1. Attardi B, Tsujii T, Friedman R, Zeng Z, Roberts JL, Dellovade T, et al. Glucocorticoid repression of gonadotropin-releasing hormone gene expression and secretion in morphologically distinct subpopulations of GT1-7 cells. Mol Cell Endocrinol 1997; 131: 241-255.

2. Selleck MA, Scherson TY, Bronner-Fraser M. Origins of neural crest cell diversity. Dev Biol 1993; 159: 1-11.

3. Anderson DJ. Cell fate determination in the peripheral nervous system: the sympathoadrenal progenitor. J Neurobiol 1993; 24: 185198.

4. Stemple DL, Anderson DJ. Lineage diversification of the neural crest: in vitro investigations. Dev Biol 1993; 159: 12-23.

5. Cooper MJ, Hutchins GM, Cohen PS, Helman LJ, Mennie RJ, Israel MA. Human neuroblastoma tumor cell lines correspond to the arrested differentiation of chromaffin adrenal medullary neuroblasts. Cell Growth Differ 1990; 1: 149-159.

6. Glick RD, Medary I, Aronson DC, Scotto KW, Swendeman SL, La Quaglia MP. The effects of serum depletion and dexamethasone on growth and differentiation of human neuroblastoma cell lines. $J$ Pediatr Surg 2000; 35: 465-472.

7. Ross S, Fischer A, Unsicker K. Sympathoadrenal progenitors in embryonic chick sympathetic ganglia show distinct responses to glucocorticoid hormones. J Neurocytol 1995; 24: 247-256.

8. Antonow-Schlorke I, Schwab M, Li C, Nathanielsz PW. Glucocorticoid exposure at the dose used clinically alters cytoskeletal proteins and presynaptic terminals in the fetal baboon brain. J Physiol 2003; 547: 117-123.

9. Romero IA, Radewicz K, Jubin E, Michel CC, Greenwood J, Couraud $\mathrm{PO}$, et al. Changes in cytoskeletal and tight junctional proteins correlate with decreased permeability induced by dexamethasone in cultured rat brain endothelial cells. Neurosci Lett 2003; 344: 112 116.

10. Biedler JL, Helson L, Spengler BA. Morphology and growth, tumorigenicity, and cytogenetics of human neuroblastoma cells in continuous culture. Cancer Res 1973; 33: 2643-2652.

11. Ross RA, Hein AM, Braca JA III, Spengler BA, Biedler JL, Scammell JG. Glucocorticoids induce neuroendocrine cell differentiation and increase expression of $\mathrm{N}$-myc in $\mathrm{N}$-type human neuroblastoma cells. Oncol Res 2002; 13: 87-94.
12. Vogel KS, Weston JA. The sympathoadrenal lineage in avian embryos. I. Adrenal chromaffin cells lose neuronal traits during embryogenesis. Dev Biol 1990; 139: 1-12.

13. Michelsohn AM, Anderson DJ. Changes in competence determine the timing of two sequential glucocorticoid effects on sympathoadrenal progenitors. Neuron 1992; 8: 589-604.

14. Ciccarone V, Spengler BA, Meyers MB, Biedler JL, Ross RA. Phenotypic diversification in human neuroblastoma cells: expression of distinct neural crest lineages. Cancer Res 1989; 49: 219-225.

15. Ross RA, Biedler JL. Presence and regulation of tyrosinase activity in human neuroblastoma cell variants in vitro. Cancer Res 1985; 45: 1628-1632.

16. Williams LR, Sandquist D, Black AC Jr, Williams TH. Glucocorticoids increase tyrosine hydroxylase activity in cultured murine neuroblastoma. J Neurochem 1981; 36: 2057-2062.

17. Tank AW, Weiner N. Induction of tyrosine hydroxylase by glucocorticoids in mouse neuroblastoma cells. Enhancement of the induction by cyclic AMP. Mol Pharmacol 1982; 22: 421-430.

18. Bradford MM. A rapid and sensitive method for the quantitation of microgram quantities of protein utilizing the principle of protein-dye binding. Anal Biochem 1976; 72: 248-254.

19. Munson PJ, Rodbard D. Ligand: a versatile computerized approach for characterization of ligand-binding systems. Anal Biochem 1980; 107: 220-239.

20. Forray C, Richelson E. Glucocorticoids potentiate the prostaglandin E1-mediated cyclic AMP formation by a cultured murine neuroblastoma clone. J Neurochem 1985; 45: 79-85.

21. Maggi R, Pimpinelli F, Molteni L, Milani M, Martini L, Piva F. Immortalized luteinizing hormone-releasing hormone neurons show a different migratory activity in vitro. Endocrinology 2000; 141: 21052112.

22. Helman LJ, Thiele CJ, Linehan WM, Nelkin BD, Baylin SB, Israel MA. Molecular markers of neuroendocrine development and evidence of environmental regulation. Proc Natl Acad Sci USA 1987; 84: 2336-2339.

23. Dunnet CW. A multiple comparison procedure for comparing several treatments with a control. J Am Stat Assoc 1955; 50: 1096 1121. 
24. Schneider N, Lanz S, Ramer R, Schaefer D, Goppelt-Struebe M. Up-regulation of cyclooxygenase-1 in neuroblastoma cell lines by retinoic acid and corticosteroids. J Neurochem 2001; 77: 416-424.

25. Hery M, Semont A, Fache MP, Faudon M, Hery F. The effects of serotonin on glucocorticoid receptor binding in rat raphe nuclei and hippocampal cells in culture. J Neurochem 2000; 74: 406-413.

26. Zhou J, Cidlowski JA. The human glucocorticoid receptor: one gene, multiple proteins and diverse responses. Steroids 2005; 70: 407417.

27. Shiratsuchi T, Ishibashi H, Shirasuna K. Inhibition of epidermal growth factor-induced invasion by dexamethasone and AP-1 decoy in human squamous cell carcinoma cell lines. J Cell Physiol 2002; 193: 340-348.

28. Delbrouck C, Doyen I, Belot N, Decaestecker C, Ghanooni R, de Lavareille A, et al. Galectin-1 is overexpressed in nasal polyps under budesonide and inhibits eosinophil migration. Lab Invest 2002; 82: $147-158$

29. Demir N, Demir R. Effects of maternal bilateral adrenalectomy on fetal rat cerebral cortex. Int J Neurosci 2001; 111: 21-38.

30. Abrahamsson PA, di Sant'Agnese PA. Neuroendocrine cells in the human prostate gland. J Androl 1993; 14: 307-309.

31. Semont A, Fache M, Hery F, Faudon M, Youssouf F, Hery M. Regulation of central corticosteroid receptors following short-term activation of serotonin transmission by 5-hydroxy-L-tryptophan or fluoxetine. J Neuroendocrinol 2000; 12: 736-744.

32. Blackhurst G, McElroy PK, Fraser R, Swan RL, Connell JM. Seasonal variation in glucocorticoid receptor binding characteristics in human mononuclear leucocytes. Clin Endocrinol 2001; 55: 683-688.

33. Gould E, Woolley CS, McEwen BS. Adrenal steroids regulate post- natal development of the rat dentate gyrus: I. Effects of glucocorticoids on cell death. J Comp Neurol 1991; 313: 479-485.

34. Trejo JL, Cuchillo I, Machin C, Rua C. Maternal adrenalectomy at the early onset of gestation impairs the postnatal development of the rat hippocampal formation: effects on cell numbers and differentiation, connectivity and calbindin-D28k immunoreactivity. J Neurosci Res 2000; 62: 644-667.

35. Herrlinger U, Schabet M, Eichhorn M, Petersen D, Grote EH, Meyermann R, et al. Prolonged corticosteroid-induced remission in primary central nervous system lymphoma: report of a case and review of the literature. Eur Neurol 1996; 36: 241-243.

36. Gut P, Huber K, Lohr J, Bruhl B, Oberle S, Treier M, et al. Lack of an adrenal cortex in Sf1 mutant mice is compatible with the generation and differentiation of chromaffin cells. Development 2005; 132: 46114619.

37. Hall A. Rho GTPases and the actin cytoskeleton. Science 1998 279: 509-514.

38. Koukouritaki SB, Gravanis A, Stournaras C. Tyrosine phosphorylation of focal adhesion kinase and paxillin regulates the signaling mechanism of the rapid nongenomic action of dexamethasone on actin cytoskeleton. Mol Med 1999; 5: 731-742.

39. Miki H, Mio T, Nagai S, Hoshino Y, Tsutsumi T, Mikuniya T, et al. Glucocorticoid-induced contractility and F-actin content of human lung fibroblasts in three-dimensional culture. Am J Physiol 2000; 278: L13-L18.

40. Clark AF, Brotchie D, Read AT, Hellberg P, English-Wright S, Pang $\mathrm{IH}$, et al. Dexamethasone alters F-actin architecture and promotes cross-linked actin network formation in human trabecular meshwork tissue. Cell Motil Cytoskeleton 2005; 60: 83-95. 